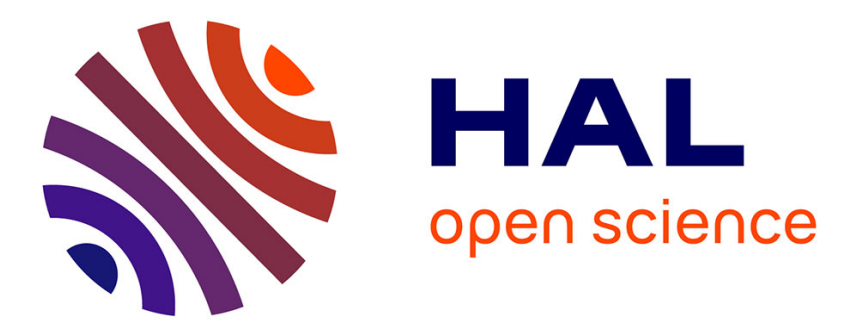

\title{
Finite element analysis of the grain size effect on diffusion in polycrystalline materials
}

Victor Lacaille, C. Morel, Eric Feulvarch, Guillaume Kermouche, Jean-Michel Bergheau

\section{- To cite this version:}

Victor Lacaille, C. Morel, Eric Feulvarch, Guillaume Kermouche, Jean-Michel Bergheau. Finite element analysis of the grain size effect on diffusion in polycrystalline materials. Computational Materials Science, 2014, 95, pp.187-191. 10.1016/j.commatsci.2014.07.026 . emse-01498591

\section{HAL Id: emse-01498591 \\ https://hal-emse.ccsd.cnrs.fr/emse-01498591}

Submitted on 13 Sep 2021

HAL is a multi-disciplinary open access archive for the deposit and dissemination of scientific research documents, whether they are published or not. The documents may come from teaching and research institutions in France or abroad, or from public or private research centers.
L'archive ouverte pluridisciplinaire HAL, est destinée au dépôt et à la diffusion de documents scientifiques de niveau recherche, publiés ou non, émanant des établissements d'enseignement et de recherche français ou étrangers, des laboratoires publics ou privés. 


\title{
Finite element analysis of the grain size effect on diffusion in polycrystalline materials
}

\author{
V. Lacaille ${ }^{\mathrm{a}, \mathrm{b}, \mathrm{c}, *}, \mathrm{C}$ Morel $^{\mathrm{a}}$, E. Feulvarch ${ }^{\mathrm{b}}$, G. Kermouche ${ }^{\mathrm{c}}$, J.-M. Bergheau ${ }^{\mathrm{b}}$ \\ ${ }^{a}$ Winoa, 528 avenue de Savoie, BP3, 38570 Le Cheylas, France \\ ${ }^{\mathrm{b}}$ Univ. Lyon, ENISE, LTDS, UMR 5513 CNRS, 58 rue Jean Parot, 42023 Saint-Etienne Cedex 2, France \\ 'École des Mines de Saint-Etienne, Science of Materials and Structures (SMS) Division, LGF UMR 5307 CNRS, 158 Cours Fauriel, 42023 Saint-Etienne, France
}

Polycrystalline materials with refined grain size are well known to have enhanced diffusion properties compared to coarse grain materials. Due to their high grain boundary density, the macroscopic diffusivity of such materials is increased. Indeed, grain boundaries are fast diffusion channels in the material. In this paper, a numerical method to calculate the diffusivity of polycrystalline materials as a function of their grain size is proposed. A homogenization technique is applied on polycrystalline representative volume elements on which diffusion calculations are performed with a finite element method. This technique allows to extract the effective diffusivity of the material for different grain sizes. A relationship is then built between the diffusivity and the grain size. It is shown that the extracted diffusivity follows a mix-ture law of both diffusivities in the grains and grain boundaries, as proposed by Hart [14], but taking into account grain boundaries randomly oriented compared to the diffusion direction.

\section{Introduction}

In the recent years, surface nanostructured materials are of great interest to enhance mechanical parts. Regarding their mechanical properties, it has been shown that they provide improved hardness, fatigue resistance and wear resistance [1-3]. They also improve the efficiency of thermochemical treatments such as nitriding by increasing the hardness [4-8] and the nitrogen penetration depth $[5,6,9]$. Moreover, the nitriding temperature $[5,7,8]$ is reduced. Likewise, the friction coefficient, the wear [48] and the corrosion [5] are also reduced. The main explanation given for a such efficiency improvement is that grain boundaries act like fast diffusion channels. Then, when the grain size decreases, the grain boundaries multiply, as well as the fast diffusion channels. Thermochemical treatments are usually described by the diffusion equation, which is a combination of both Fick's laws:

$\frac{\partial c}{\partial t}=\operatorname{div}(\overrightarrow{\operatorname{Drad}}(c))$

where $c(\vec{x}, t)$ is the concentration of the diffusing element at point $\vec{x}$ and time $t$, and $D$ is its diffusivity in the material. In this formulation, no thermodynamic interactions are taken into account and

* Corresponding author at: Winoa, 528 avenue de Savoie, BP3, 38570 Le Cheylas, France. one diffusing element is considered. The term $D$ is then a macroscopic value of the diffusivity which takes into account both diffusion processes in grains and along grain boundaries. At a smaller scale, these processes can be separated in two single diffusion processes occurring in two different media with different diffusivities: $D_{g}$ for the diffusion inside the grains and $D_{g b}$ for the diffusion along the grain boundaries. It is possible to find some numerical values for diffusivities of both grains and grain boundaries in the case of selfdiffusion [10-12]. Table 1 gives an example of both grain and grain boundary diffusivities for aluminum in the case of self diffusion at $400{ }^{\circ} \mathrm{C}$. According to Harrison's classification [13], in the case of type A diffusion kinetics, Hart has proposed an analytic expression for the effective diffusivity [14] with grain boundaries parallels to the diffusion direction.

$D=f_{v} D_{g b}+\left(1-f_{v}\right) D_{g}$

This relations is similar to the Wiener upper bound for the real effective permittivity in multicomponent media [15] and to the upper Voigt bound in elasticity [16]. $f_{v}$ is the volume fraction of grain boundaries and $D$ the effective diffusivity which can be used in the diffusion Eq. (1). The volume fraction of grain boundaries $f_{v}$ can also be written

$f_{v}=\beta \frac{\delta}{d}$ 
Table 1

Aluminum self diffusion coefficients at $400{ }^{\circ} \mathrm{C}$ for grains and grain boundaries [10].

\begin{tabular}{lll}
\hline & Brown and Ashby [11] & Gust et al. [12] \\
\hline$D_{g}\left(\mathrm{~mm}^{2} / \mathrm{s}\right)$ & $1.8 \times 10^{-11}$ & $2.33 \times 10^{-11}$ \\
$D_{g b}\left(\mathrm{~mm}^{2} / \mathrm{s}\right)$ & $1.84 \times 10^{-5}$ & $6.27 \times 10^{-5}$ \\
\hline
\end{tabular}

where $\delta$ is the grain boundary thickness and $d$ is the grain size. $\beta$ is a numerical factor depending on the grain shape. Eq. (2) was extended to the Hart-Mortlock equation [17] which takes into account segregation at grain boundaries. Kalnins et al. [18] has also developed a model which describes diffusion in a two-phase material with segregation effects. The combination of these two equations has been performed by Belova and Murch [19] to build an analytical model of the effective diffusivity in a 2D cubic shaped polycrystal. Also, some authors studied the grain boundary diffusion effect on the effective diffusivity with numerical methods. Gryaznov et al. [20] and Bassman et al. [21] have made a finite element analysis considering two different media: grains and grain boundaries. This type of methodology has also been applied with the finite difference method [22] or by computation of the effective conductivity of two dimensional disordered random Voronoi networks [23] with Kirchhoff equations resolved by Lanczos algorithm [24]. The aim of this work is to expand Hart's equation to a 3D media where the grain boundaries are randomly oriented regarding the diffusion direction with help of an homogenization technique compatible with representative volume elements (RVE) as shown in Fig. 1. This technique enables to extract the effective diffusivity of the considered RVE. The second step of this work stands on a numerical study of flux dissociation in grains and along grain boundaries. It will lead to formulate the effective diffusivity as a weighted arithmetic average as expressed by Hart. The ponderation coefficient of this expression will be numerically measured on multiple representative volume elements and linked to the grain size.

\section{Homogenization technique}

To calculate the effective diffusivity in general cases, it is not possible to take the average of the different local diffusivities as

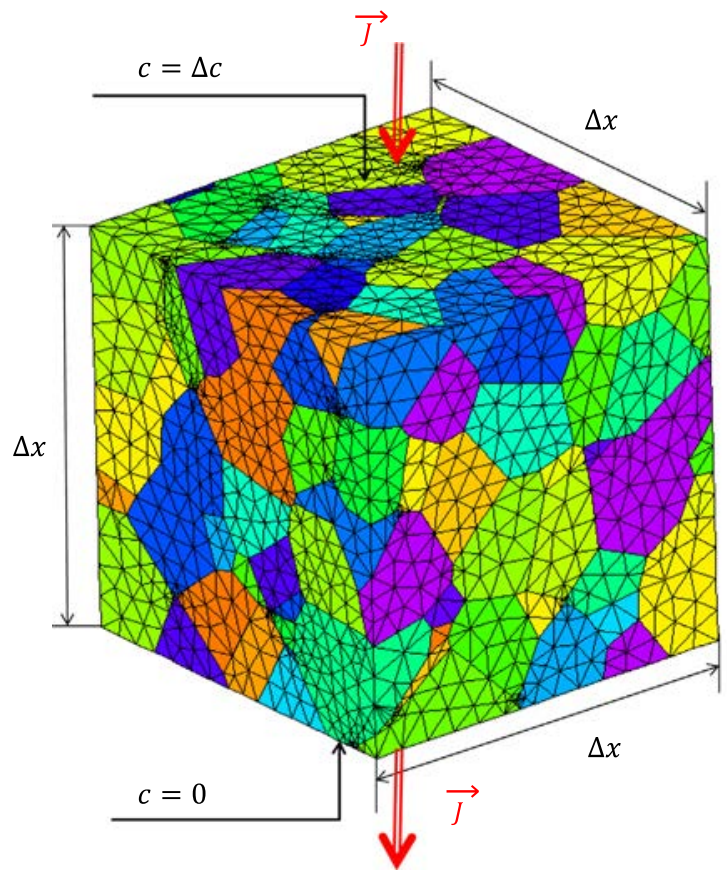

Fig. 1. Example of a representative volume element with 250 grains generated by the software Neper [25]. in perfect interfaces described by Hart's model. The homogenization technique presented here aims at giving a method to extract an effective diffusivity $D$ from a diffusion simulation on a cubic RVE by means of the finite element method. Let us consider a cubic RVE of $\Delta x$ square under a concentration difference $\Delta c$ on two of its opposite faces. This RVE can be considered as a heterogeneous material constituted of grains and grain boundaries. Because the concentration is prescribed on two opposite faces of the cube, the diffusion occurs in the direction perpendicular to these surfaces. Let $x$ designing the considered point on this direction. At a local scale, the flux density through the RVE is given by

$\vec{J}=-D(x) \frac{\partial c(x)}{\partial x}$

where $D(x)$ can be either $D_{g}$ or $D_{g b}$. Then at steady state, considering this cube as a homogeneous of diffusivity $D$, the concentration gradient along the RVE can be expressed as the concentration slope

$\frac{\partial c(x)}{\partial x} \approx\left\langle\frac{\partial c(x)}{\partial x}\right\rangle=\frac{\Delta c}{\Delta x}$

where the brackets denote a global average on the whole RVE. Then, by integrating the flux density on the whole incoming or outcoming surface of the RVE, where it is assumed to be constant due to the homogenization hypothesis, the total flux $\Phi$ is given by

$\Phi=-D \frac{\Delta c}{\Delta x} S$

with $S=\Delta x^{2}$ is the section of the cube perpendicular to the diffusion direction. The diffusivity is then linked to the concentration difference $\Delta c$, to the side of the cube $\Delta x$ and to the flux of chemical element $\Phi$ which is easily measurable from a finite element calculation. The effective diffusivity can be deduced by performing a diffusion simulation on a cubic RVE under a concentration difference at steady state and by measuring the flux on one of its two surfaces where it passes through.

$D=\frac{-\Phi}{\Delta x \Delta c}$

\section{Finite elements model}

\subsection{Generation of representative volume elements with equiaxed grains}

To generate the cubic RVEs, the software Neper [25] has been used. This software enables to generate such RVEs by adjusting the side of the cube $\Delta x$ and the number of grains in the cube $N$ so the grain size can not be set directly. The grain shapes are obtained with a Voronoï tesselation. These RVEs are produced as a mesh of the grain and the grain boundaries, where grains are meshed with 3D elements and grain boundaries are meshed with 2D elements.

\subsection{Diffusion model}

To simulate the diffusion at steady state, calculations are performed with the software Sysweld ${ }^{\circledR}$ [26]. The problem is solved using Eq. (1) and two different diffusivities, $D_{g}$ and $D_{g b}$ are affected to grains and grain boundaries respectively. A thickness $\delta$ is affected to the grain boundaries in order to model the volumic diffusion along them. The grains are considered to be equiaxed, due to their generation method. Their crystallographic orientation is not taken into account. 


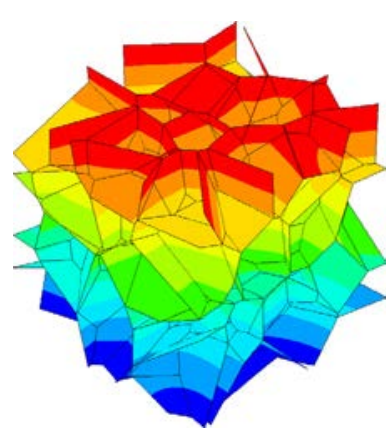

(a) Voronoi 3D

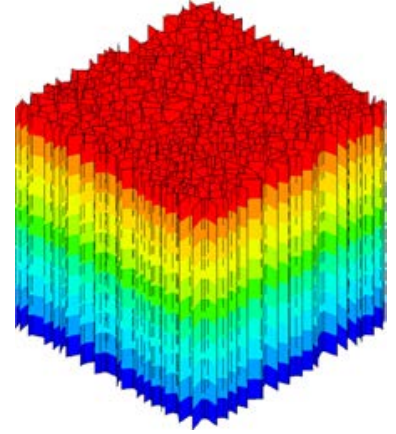

(b) Columnar

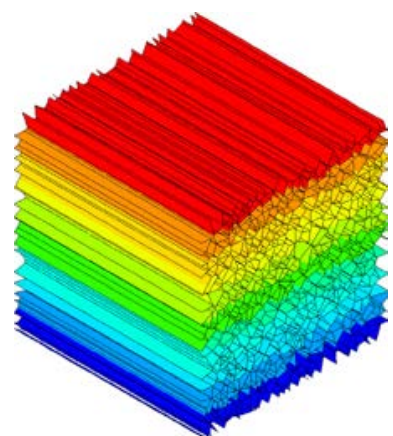

(c) Voronoi 2D

Fig. 2. Grain shapes used to perform simulations. Diffusion direction is vertical, highlighted by the color gradient. (For interpretation of the references to colour in this figure legend, the reader is referred to the web version of this article.)

\subsection{Set of representative volume elements}

150 different RVEs have been created, with a cubic shape with $\Delta x=1 \mathrm{~mm}$. The grain numbers are set between 50 and 500 grains by steps of 50 grains. For one number of grains, 5 different RVEs have been generated. These methodology has been repeated for 3 different grain shapes:

1. The grains are generated with a 3D Voronoï tesselation, which is the default behavior of Neper (see Fig. 2a).

2. The grains are generated with a 2D Voronoï tesselation in order to create columnar grains in the direction of the diffusion (see Fig. 2b).

3. The grains are generated with a 2D Voronoï tesselation in order to create columnar grains perpendicular to the diffusion direction, which is equivalent to a planar 2D Voronoï tesselation (see Fig. 2c).

The concentration difference $\Delta c$ was set to $100 \%$.

\section{Flux dissociation in a representative volume element}

In this part three cases of diffusion are studied.

1. The whole RVE is subject to diffusion: $D_{g}=1 \mathrm{~mm}^{2} / \mathrm{s}, D_{g b}=10^{6} \mathrm{~mm}^{2} / \mathrm{s}$ and $\delta=10^{-6} \mathrm{~mm}$.

2. Only the grain boundaries are subject to diffusion: $D_{g}=0, D_{g b}=10^{6} \mathrm{~mm}^{2} / \mathrm{s}$ and $\delta=10^{-6} \mathrm{~mm}$.

3. Only the grains are subject to diffusion: $D_{g}=1 \mathrm{~mm}^{2} / \mathrm{s}$ and $D_{g b}=0$.

The third case described above is equivalent to a homogeneous cube. Indeed, the grain boundaries are meshed with surface ele- ments. The nodes composing a grain boundary belong to this grain boundary and to both grains toward the grain boundary. If the chemical element cannot diffuse through the grain boundaries $\left(D_{g b}=0\right)$, they can still diffuse through the grains because there is at least one node linking a grain to its neighbor. Regarding the diffusivities presented in Table 1 , the grain boundary diffusivity has been set 1 million times higher than the grain diffusivity. Let us consider $\Phi_{g b}$ as the flux in the surface mesh of grain boundaries only. And let us consider $\Phi_{g}$ as the flux in the RVE without grain boundaries, which is the flux in a homogeneous cube. They respectively correspond to the measurement of $\Phi$ in the second and third case of diffusion described above. Thus, in order to have $\Phi_{g}$ and $\Phi_{g b}$ of the same order of magnitude, the grain boundary thickness $\delta$ has been set to $10^{-6} \mathrm{~mm}$ i.e. 1 million times lower. This value is consistent with experimental values obtained by high-resolution transmission microscopy, field ion microscopy and other techniques [27-30].

\subsection{Results and discussion}

In Fig. 3 the relative difference $\varepsilon=\left|\frac{\Phi-\Phi_{g}-\Phi_{g b}}{\Phi}\right|$ is plotted versus the number of grains $N$ in the case of Voronoï 3D grain shapes (Fig. 2a). This quantity is representative of the ability of both flux $\Phi_{g}$ and $\Phi_{g b}$ to be taken separately and added together in order to calculate the real total flux $\Phi$.

It appears that above 50 grains the relative difference $\varepsilon$ remains less than $2 \%$. It means that the following assumption can be made

$\Phi \approx \Phi_{g}+\Phi_{g b}$

Then, 50 grains is a low limit for the flux dissociation hypothesis and for the volume element representativeness. Moreover, the flux in a homogeneous cube of diffusivity $D_{g}$ is already known

$\Phi_{g}=-D_{g} \Delta x \Delta c$

Hence, by combining Eqs. (7)-(9) it becomes

$D=\left(D_{g}-\frac{\Phi_{g b}}{\Delta c \Delta x}\right)$

This equation shows that the study of the effective diffusivity stands on the measurement of the flux in grain boundaries only. Then the calculations can be performed on the grain boundaries only instead of being performed on the whole representative volume element. The surface mesh of the grain boundaries is enough

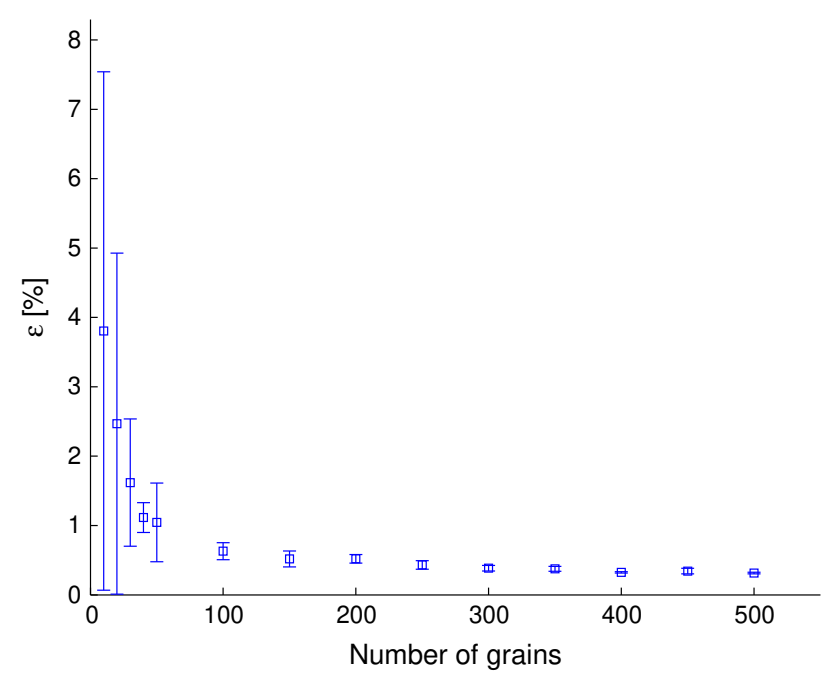

Fig. 3. Validity criterion of the flux superposition hypothesis. 


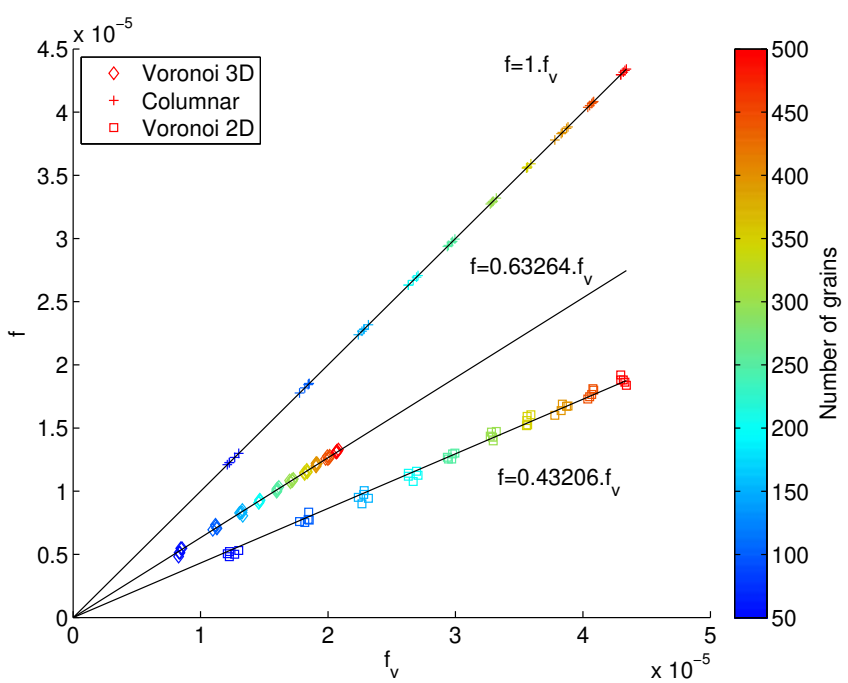

Fig. 4. Weighting coefficient of the homogeneous diffusivity as a function of the volume fraction of grain boundary.

to know the whole flux in the RVE, which saves some calculation time. The flux in a set of grain boundaries is written as follows

$\Phi_{g b}=\int_{S_{g b}} \vec{J} \cdot \vec{n} d S$

where $S_{g b}$ is the surface in the grain boundary set where the flux comes in (or out) and $\vec{n}$ its normal unit vector. By extracting the grain boundary thickness $\delta$ it can also be written

$\Phi_{g b}=\delta D_{g b} \int_{l_{g b}}-\overrightarrow{\operatorname{grad}}(c) \cdot \vec{n} d l$

where $l_{g b}$ is the grain boundary length corresponding to $S_{g b}$ and the term $\overrightarrow{\operatorname{grad}}(c) \cdot \vec{n}$ is representative of the grain boundary orientation: if the macroscopic diffusion direction - marked by the vector $\vec{n}$ - is perpendicular to the grain boundary - marked by the vector $\overrightarrow{\operatorname{grad}}(c)-$, there will be no flux through it. According to the problem linearity, the flux is proportional to the prescribed concentration difference $\Delta c$. It leads to the following expression

$\Phi_{g b}=-D_{g b} \delta \Delta c \alpha$

where $\alpha$ is a scalar. Thus, by extracting the side of the cube, it becomes

$\Phi_{g b}=-D_{g b} \delta \frac{\Delta c}{\Delta x} l_{e q}$

where $l_{e q}$ represents an equivalent length of flux transit. By combining Eqs. (7), (8), (9) and (14), it becomes:

$D=f D_{g b}+(1-f) D_{g}$

where

$f=\frac{\delta l_{e q}}{\Delta x^{2}+\delta l_{e q}}$

This equation is closely related to Hart's equation but is applicable on 3D structures with non columnar grains. The following part aims at expressing the weighting coefficient $f$ as a function of the grain size $d$.

\section{Effective diffusivity model}

\subsection{Results}

According to Hart's equation, the weighting coefficient $f$ of Eq. (15) should be equal to $f_{v}$ the volume fraction of grain boundaries.
For the whole set of RVEs, both $f$ and $f_{v}$ values are extracted and plotted in Fig. 4. $f$ is extracted from a combination of Eqs. (14) and (16) by measuring $\Phi_{g b}$ and $f_{v}$ is obtained with the following relation:

$f_{v}=\frac{V_{g b}}{V_{g b}+V_{g}}=\frac{S_{v} \delta \Delta x^{3}}{S_{v} \delta \Delta x^{3}+\Delta x^{3}}=\frac{S_{v} \delta}{S_{v} \delta+1}$

where $S_{v}$ is the grain boundary surface per unit volume in the RVE, which can be numerically measured on the mesh. It also corresponds to the grain boundary density. It appears that the weighting coefficient $f$ is directly proportional to the volume fraction of grain boundaries $f_{v}$. Let us call $q$ the proportionality coefficient, it changes with the grain shape but remains less than one, except in the case of columnar grains in the diffusion direction where it is equal to one.

\subsection{Discussion}

The proportionality between $f$ and $f_{v}$ can then be written

$f=q f_{v}$

Table 2 summarizes the different values of $q$ obtained in the 3 cases. For a given value of $q$, the model predicts the effective diffusivity as a function of the grain boundary volume fraction. $q$ seems to depend on the grain shape and to be representative of the grain boundary fraction participating to diffusion. In the case of columnar grains, the model becomes equivalent to Hart's Eq. (2). In this case, the whole grain boundary surface participates to diffusion and $q=1$. For this configuration, the calculation of $q$ would have been trivial but the aim is to highlight that its numerical measurement is consistent with this well-known solution. The link with the grain size can be established through the grain boundary density $S_{v}$, which requires to make an hypothesis on the grain shape. For example for spherical grains of diameter $d$, this grain boundary surface per unit volume can be obtained by dividing the surface of the sphere by the double of its volume, because a grain boundary belongs to two grains.

$S_{v}=\frac{4 \pi\left(\frac{d}{2}\right)^{2}}{2 \times \frac{4}{3} \pi\left(\frac{d}{2}\right)^{3}}=\frac{3}{d}$

A similar approach for other grain shapes leads to a general expression for the grain boundary density:

$S_{v}=\frac{\beta}{d}$

where $\beta$ depends on the grain shape and $d$ is the grain size. The definition of the grain size can vary with the grain shape. In our cases, we chose to assume the grains as spherical for the Voronoi 3D configuration because they do not present any preferential direction. For the same reason, in both columnar and Voronoi 2D cases, we assumed the grains as cylindrical. In these 3 cases, the grain size is defined as the diameter of the grains. The value $\beta$ is a link between the grain size and the volume (or surface) fraction of grain boundaries. It can be obtained by measuring the grain boundary

Table 2

Grain Shape parameters $q$ and $\beta$ for the 3 different shapes studied.

\begin{tabular}{lrl}
\hline Case & $q$ & $\beta$ \\
\hline Voronoi 3D & 0.63264 & $3.1685 \pm 0.13$ \\
Columnar & 1 & $2.1455 \pm 0.06$ \\
Voronoi 2D & 0.43206 & $2.1455 \pm 0.06$ \\
\hline
\end{tabular}


density on these multiple grain shapes. Then, the grain size can be extracted with these relations

$$
\begin{aligned}
& d=2 \sqrt[3]{\frac{3 \Delta x^{3}}{4 \pi N}} \text { For spherical grains } \\
& d=2 \sqrt{\frac{\Delta x^{2}}{\pi N}} \text { For cylindrical grains }
\end{aligned}
$$

A linear regression lead to estimate $\beta$ with the least squares method for each grain shape. Table 2 shows its values for the different grain shapes studied. The relation (20) can also be obtained by dividing the volume fraction of grain boundaries of Eq. (3) by $\delta$. Considering $S_{v} \delta$ to be negligible compared to 1 , the volume fraction can also be obtained with Eq. (17). Hence, replacing all parameters with Eqs. (15), (17) and (18), the effective diffusivity model can be written as follows

$D=D_{g}+q \frac{\beta \delta}{d+\beta \delta}\left(D_{g b}-D_{g}\right)$

This model is valid when the contrast between grain boundary diffusivity and lattice diffusivity is high and when the grain boundary thickness is very low compared to the grain size, in order to respect the hypothesis that grain boundaries can be represented by surface mesh elements. Thus, considering that $\beta \delta$ is negligible compared to $d$, and that $D_{g b} \gg D_{g}$, Eq. (22) becomes

$D=D_{g}+q \beta \frac{\delta}{d} D_{g b}$

The main difference with the Hart Eq. (2) can be found in this factor $q$ which takes into account grain boundaries not parallel or perpendicular to the diffusion direction. With a global point of view, there are two geometrical parameters in this model: $\beta$ and $q$. The value of $q$ is representative of the grain boundaries mean orientation compared to the diffusion direction. The more grain boundary surface containing the diffusion direction there will be, the more $q$ will be close to unity. And by increasing the proportion of grain boundaries perpendicular to the diffusion direction, the value of $q$ decreases. The difference between these two parameters is clearly underlined in Table 2: for both cases Columnar and Voronoi $2 D$, the grain shape remains the same just as $\beta$. However, $q$ takes different values, following the grains orientation.

\section{Conclusion}

In this paper, a homogenization technique is proposed to find the effective diffusivity of polycrystalline media, using the finite elements method on cubic representative volume elements, where grain boundaries are meshed with 2D elements. A criterion has been highlighted for the flows dissociation which is a criterion for the volume element representativeness. The flux dissociation enables to expand the Hart model to a 3D representative volume element. Then, an analytic formulation of the relationship between diffusivity and grain size has been proposed from the study of RVEs with different grain sizes. It constitutes an improvement of Hart's equation by taking into account grain boundaries in random directions.

For the future studies, the focus will be given to the meaning of the $q$ parameter, which seems to depends on the grain geometry. The method will be applied on representative volume elements with non equiaxed grains and the value of $q$ will be studied as a function of the grain shape or the grain geometrical orientation.

A more general aim of this study is to provide an engineering tool to determine the effective diffusivity for a given microstructure. Thus, the homogenization method presented here is expandable to any transport phenomenon like thermal studies for the calculation of an effective thermal conductivity for example.

\section{References}

[1] K. Lu, J. Lu, Mater. Sci. Eng. A 375-377 (2004) 38-45.

[2] T. Roland, D. Retraint, K. Lu, J. Lu, Scr. Mater. 54 (2006) 1949-1954.

[3] N. Tao, Z. Wang, W. Tong, M. Sui, J. Lu, K. Lu, Acta Mater. 50 (2002) 4603-4616.

[4] Y. Lin, J. Lu, L. Wang, T. Xu, Q. Xue, Acta Mater. 54 (2006) 5599-5605.

[5] W. Tong, N. Tao, Z. Wang, J. Lu, K. Lu, Science 299 (2003) 686-688.

[6] W. Tong, C. Liu, W. Wang, N. Tao, Z. Wang, L. Zuo, J. He, Scr. Mater. 57 (2007) $533-536$

[7] W. Tong, Z. Han, L. Wang, J. Lu, K. Lu, Surf. Coat. Technol. 202 (2008) 49574963.

[8] H. Zhang, L. Wang, Z. Hei, G. Liu, J. Lu, K. Lu, Z. Metallkd. 10 (2003) 1143-1147.

[9] J. Gu, D. Bei, J. Pan, J. Lu, K. Lu, Mater. Lett. 340 (2002) 340-343.

[10] C. Herzig, Y. Mishin, Diffusion in Condensed Matter, Springer, 2005. pp. 337366 (Chapter 8).

[11] A.M. Brown, M.F. Ashby, Acta Metall. 28 (1980) 1085-1101.

[12] W. Gust, S. Mayer, A. Boegel, B. Predel, Generalized representation of grain boundary self-diffusion, in: Journal de Physique Colloques, vol. 46, 1985, pp. C4-537-C4-544.

[13] L.G. Harrison, Trans. Faraday Soc. 57 (1961) 1191-1199.

[14] E. Hart, Acta Metall. 5 (10) (1957) 597.

[15] O. Wiener, Abh. Math. Phys. K1 Königl. Sächs. Ges. 32 (1912) 509.

[16] W. Voigt, Ueber die beziehung zwischen den beiden elasticitätsconstanten isotroper körper 274 (1889) 573.

[17] A. Mortlock, Acta Metall. 8 (2) (1960) 132-134.

[18] R.D. Kalnins, L. Markosian, B.J. Meier, M.A. Kowalski, J.C. Lee, P.L. Davidson, M. Webb, J.F. Hughes, A. Finkelstein, WYSIWYG NPR: drawing strokes directly on 3D models, ACM Transactions on Graphics (TOG), vol. 21, ACM, 2002, pp. 755762 .

[19] I. Belova, G. Murch, J. Phys. Chem. Solids 64 (5) (2003) 873-878.

[20] D. Gryaznov, J. Fleig, J. Maier, Solid State Sci. 10 (2008) 754-760.

[21] L. Bassman, N. Ibrahim, P. Pinsky, K. Saraswat, M. Deal, Mesoscale modeling of diffusion in polycrystalline structures, in: 1997 International Conference on Simulation of Semiconductor Processes and Devices, 1997. SISPAD '97, 1997, pp. 149-152.

[22] N. Yazdipour, A. Haq, K. Muzaka, E. Pereloma, Comput. Mater. Sci. 56 (0) (2012) $49-57$.

[23] A. Priolo, H. Jaeger, A. Dammers, S. Radelaar, Phys. Rev. B 46 (22) (1992) 14889.

[24] C. Lanczos, Nat. Bur. Std. 45 (255) (1950) 49.

[25] R. Quey, P. Dawson, F. Barbe, Comput. Methods Appl. Mech. Eng. 200 (17-20) (2011) 1729-1745.

[26] ESI-Group, User's Manual, 2014.

[27] I. Kaur, Y. Mishin, W. Gust, Fundamentals of Grain and Interphase Boundary Diffusion, Wiley, 1995

[28] A. Sutton, R. Balluffi, Interfaces in Crystalline Materials, Monographs on the Physics And Chemistry of Materials, OUP Oxford, 1995.

[29] D. Wolf, S. Yip, Materials Interfaces: Atomic-level Structure and Properties, Springer, 1992

[30] J. Howe, Interfaces in materials: atomic structure, thermodynamics and kinetics of solid-vapor, solid-liquid and solid-solid interfaces, A WileyInterscience publication, Wiley, 1997. 